\title{
Treatment patterns of in-patient spasticity medication use after traumatic spinal cord injury: a prospective cohort study
}

\author{
Kaila A. Holtz ${ }^{1}$ - Elena Szefer ${ }^{2}$ - Vanessa K Noonan ${ }^{3}$ - Brian K. Kwon ${ }^{4,5} \cdot$ Patricia B. Mills $^{1,4,6}$
}

Received: 26 February 2018 / Revised: 16 May 2018 / Accepted: 21 May 2018 / Published online: 14 June 2018

(c) International Spinal Cord Society 2018

\begin{abstract}
Study Design Prospective cohort study using the Rick Hansen SCI Registry (RHSCIR) and retrospective medical chart review.

Objective To describe treatment patterns of in-patient anti-spasticity medication use following traumatic spinal cord injury (SCI) in acute and rehabilitation hospital settings in British Columbia, Canada.

Setting Quaternary trauma center, rehabilitation center.

Methods Individuals with traumatic SCI between 2005 and 2014 enrolled in the Vancouver RHSCIR site $(N=917)$ were eligible for inclusion. Oral and injectable anti-spasticity medication use were the main outcome measures.

Results In 769 participants, higher neurological level and injury severity were associated with in-patient anti-spasticity medication use ( $p<0.001$ for both). Of individuals with cervical and thoracic injuries $(n=589), 37 \%$ were prescribed antispasticity medication during hospital admission. Baclofen was the most commonly used first line oral therapy. Mean (SD) and median time from injury to Baclofen initiation was $70(69)$ and 50 days, respectively. The probability of having initiated an in-patient anti-spasticity medication was $55 \%(95 \% \mathrm{CI}(49,60))$ for individuals 6 months post-injury, and $71 \%(95 \% \mathrm{CI}$ $(62,79))$ for individuals 12 months post-injury. At community discharge, the prevalence of oral and injectable anti-spasticity medication use was 26 and 5\%. Practice patterns of anti-spasticity medication use (2005-2009 vs. 2010-2014) have not changed significantly over time.

Conclusions This is the first large prospective cohort study of in-patient anti-spasticity medication use following traumatic SCI. Results from our study inform clinicians and individuals of "real world" anti-spasticity medication use among individuals with traumatic SCI and may help guide care for this population in the community.
\end{abstract}

Patricia B. Mills

patricia.mills@vch.ca

1 Division of Physical Medicine and Rehabilitation, Department of Medicine, University of British Columbia, Vancouver, BC, Canada

2 Emmes Canada, Burnaby, BC, Canada

3 Rick Hansen Institute, Vancouver British Columbia, Vancouver, BC, Canada

4 ICORD (International Collaboration on Repair Discoveries), University of British Columbia, Vancouver, BC, Canada

5 Vancouver Spine Surgery Institute, Department of Orthopaedics, University of British Columbia, Vancouver, BC, Canada

6 Rehabilitation Research Program, Vancouver Coastal Health Research Institute, Vancouver, BC, Canada

\section{Introduction}

Spasticity, a movement disorder characterized by intermittent or sustained uncontrolled muscle spasms [1] is a secondary complication that occurs in the majority (59-67\%) of individuals following spinal cord injury (SCI) [2-6]. The presence of spasticity can be useful, facilitating activities such as transfers [7], but it can also be problematic, requiring treatment [6]. Individuals with spasticity report difficulties across several domains [8] and are more likely to report pain, bladder problems, sleep interference, contractures, skin breakdown, difficulties with activities of daily living and lower quality of life [3, 7, 9-12].

Spasticity management may include bracing, physical therapy, therapeutic modalities such as electrical stimulation, oral and/or injectable medications, and surgery [13]. Recommended medications include Baclofen (gammaaminobutyric acid analog), benzodiazepines (e.g., 
Diazepam, gamma-aminobutyric acid agonist), Tizanidine (a2-adrenergic receptor agonist), and Dantrolene (post synaptic muscle relaxant), as well as focal chemodenervation agents botulinum toxin (e.g., Botox, Dysport, Xeomin) and phenol [14-17]. However, at least 2 systematic reviews have noted a relative paucity of high quality controlled studies demonstrating specific or comparative efficacy of these oral [18] and injectable [14] agents in the SCI population. Reporting "real-world" practice patterns will inform clinicians, provide education for individuals, and generate novel research questions.

The primary objective of this study was to describe the pattern of anti-spasticity medication use during in-patient hospitalization in a large prospective cohort of individuals with SCI. Second, we evaluated anti-spasticity medication use at community discharge, and assessed differences in anti-spasticity medication prescription patterns over time from 2005 to 2014.

\section{Methods}

\section{Study design}

This was a prospective cohort study that utilized spinal cord injured individuals enrolled at the Vancouver site of the national Rick Hansen Spinal Cord Injury Registry (RHSCIR). Medication data and selected injury characteristics were confirmed retrospectively using in-patient medical charts from the acute care and rehabilitation hospitals. Ethics approval was obtained from local Institutional Review Boards.

\section{Eligibility criteria}

All individuals at or over the age of 16 years admitted between March 2005 and March 2014 with a traumatic SCI were eligible for inclusion. Participants were excluded from analysis if they died prior to discharge or had incomplete medication records (see Fig. 1 for study diagram).

\section{RHSCIR and inpatient medical charts review}

RHSCIR is a prospective observational database of individuals with SCI admitted to major trauma and rehabilitation centers across Canada [19]. In this study, sociodemographic factors, injury characteristics, hospital admission and community discharge dates, and neurological variables were abstracted from the core RHSCIR dataset. The "core dataset" includes demographics and details of the injury and neurological impairment and was created for every patient as part of our center's standard of care. Neurological variables were obtained using the

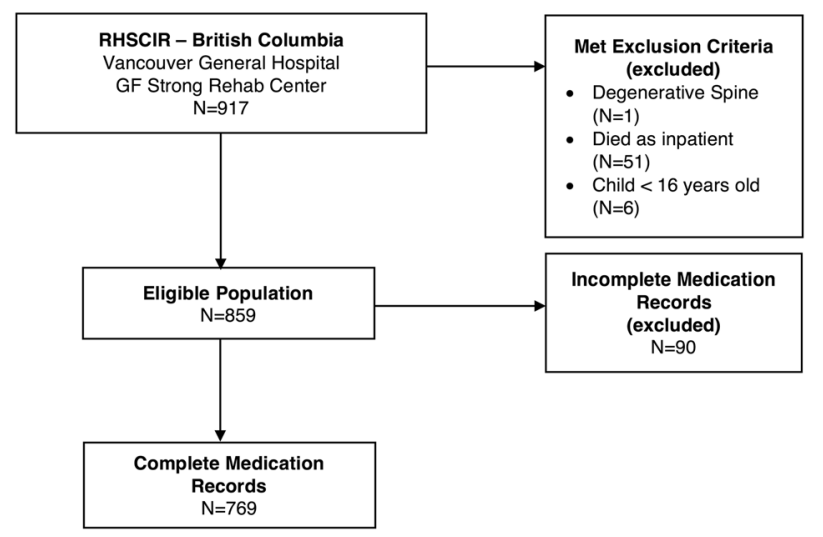

Fig. 1 Study flowchart

International Standards for Neurological Classification of Spinal Cord Injury to determine the neurologic level and American Spinal Injury Association Impairment Scale (AIS) grade [20]. Medication information was abstracted for RHSCIR participants from the inpatient medical charts. Anti-spasticity medication start date, start dose and discharge dose (totalled over $24 \mathrm{~h}$ ) was collected. Dates and dosage of focal chemodenervation with botulinum toxin or phenol for spasticity management were also abstracted. At community discharge, individuals were considered to be on anti-spasticity medication if they had one or more of: (1) an oral medication prescribed for the purpose of spasticity management (e.g., Baclofen, Tizanidine, Diazepam, Clonazepam, or Dantrolene); (2) a botulinum toxin injection for spasticity within the last 90 days $[17,21]$ or (3) a phenol injection for spasticity within the last 6 months [14].

\section{Statistical analysis}

The proportion of individuals on an anti-spasticity medication during inpatient stay (acute care and rehabilitation hospitals) and at community discharge, as well as the proportion of individuals within neurologic levels and AIS grades on anti-spasticity medications, were calculated. Chisquare tests were used to assess the association between neurologic level or AIS grade and receiving a treatment for spasticity. To test whether age or gender were associated with being discharged on an anti-spasticity medication, a likelihood-ratio test and Fisher's exact test were used to test each respective hypothesis. Clopper-Pearson 95\% confidence intervals (CI) were computed for the total proportion of individuals on medication, as well as the proportions of individuals on medication for each neurologic level and AIS grade. Tukey tests adjusted for multiple comparisons were performed to determine if there were significant differences in the proportion of individuals on medication between neurologic levels and AIS grades. Fisher's exact test was employed to compare the proportion of individuals 
within each neurologic level and AIS grade across discharge time intervals to determine whether practice patterns of prescribing any oral or injectable anti-spasticity medications were different for individuals discharged between 2005-2009 and 2010-2014. A Kaplan-Meier curve for time to initiation of spasticity medication was calculated. The relative risk of being discharged on an anti-spasticity medication was also calculated for individuals who started a medication within 6 months of injury, compared to individuals who began treatment $>6$ months after admission. The 6-month cut-off for comparing the probability of being discharged on spasticity medication was selected post-hoc after it was observed that the probability of initiating antispasticity medication after 6 months increased more slowly compared to prior to 6 months. Descriptive statistics describing the number of days to starting any oral or injectable anti-spasticity medication, for the subset of individuals who took the medications, were also performed.

\section{Results}

Figure 1 illustrates the study flowchart. $N=917$ individuals were eligible for inclusion. The cohort used in final data analysis was $N=769$. Participant characteristics are described in Table 1. Stratification of neurological levels of injury was used based on the previous work by this group studying the prevalence of spasticity after SCI [2]. Mean (SD) age was 46 (19) years. Mean (SD) time from injury to community discharge was 108 (95) days (range 1-728 days). Among the 420 individuals with known mechanism of injury, the mechanism of injury was a fall in 157 individuals (37\%), transport in 135 (32\%), sports in 92 (22\%), assault either blunt or penetrating in $17(4 \%)$, and other traumatic cause in $19(5 \%)$.

\section{Inpatient anti-spasticity medication use}

The prevalence of anti-spasticity medication use is listed in Table 2. Of all individuals, 32\% (95\% CI $(29,36)$ ) were prescribed anti-spasticity medication at some point during hospital admission. Taking anti-spasticity medication was associated with neurological injury level and AIS grade $(p<0.001)$. Anti-spasticity medication use was similar between injury severity grades A (43\%), B (49\%) and C (46\%), and significantly less in D injuries, $(23 \%), p<0.001$.

Medication start and discharge doses are listed in Table 3. The most commonly prescribed anti-spasticity medication was Baclofen. Among individuals treated with Baclofen, mean (SD) time from injury to Baclofen initiation was 70 (69) days, median time to first Baclofen dose was 50 days and it was initiated between 1 and 458 days postinjury. Baclofen was prescribed as first line treatment in
Table 1 Cohort demographics at community discharge

\begin{tabular}{|c|c|c|}
\hline & \multicolumn{2}{|c|}{ Total } \\
\hline & $N$ & $\%$ \\
\hline \multicolumn{3}{|l|}{ Age } \\
\hline $16-30$ & 211 & 27 \\
\hline $31-45$ & 171 & 22 \\
\hline $46-60$ & 191 & 25 \\
\hline $61-75$ & 142 & 18 \\
\hline $76+$ & 55 & 7 \\
\hline \multicolumn{3}{|l|}{ Sex } \\
\hline Female & 162 & 21 \\
\hline Male & 607 & 79 \\
\hline \multicolumn{3}{|c|}{ Neurological level } \\
\hline $\mathrm{C} 1-\mathrm{C} 8$ & 429 & 56 \\
\hline $\mathrm{T} 1-\mathrm{T} 12$ & 160 & 21 \\
\hline L1-S5 & 105 & 14 \\
\hline $\begin{array}{l}\text { C1-T12+ } \\
\text { L1-S5 }\end{array}$ & 7 & 1 \\
\hline Unknown & 68 & 9 \\
\hline \multicolumn{3}{|c|}{ Neurological grade (AIS) } \\
\hline A & 174 & 23 \\
\hline B & 68 & 9 \\
\hline $\mathrm{C}$ & 83 & 11 \\
\hline $\mathrm{D}$ & 360 & 47 \\
\hline $\mathrm{E}$ & 15 & 2 \\
\hline Unknown & 69 & 9 \\
\hline Total & 769 & 100 \\
\hline
\end{tabular}

Table 2 Prevalence of anti-spasticity medication use by neurological injury level and AIS grade

\begin{tabular}{|c|c|c|c|c|c|c|c|}
\hline & \multirow{2}{*}{$\begin{array}{l}\text { All } \\
N\end{array}$} & \multicolumn{3}{|c|}{$\begin{array}{l}\text { During inpatient } \\
\text { care }\end{array}$} & \multicolumn{3}{|c|}{ At discharge } \\
\hline & & $N$ & $\%$ & $95 \%$ CI & $N$ & $\%$ & $95 \%$ CI \\
\hline \multicolumn{8}{|l|}{ Neurological level } \\
\hline $\mathrm{C} 1-\mathrm{C} 8$ & 429 & 163 & 38 & $(33,43)$ & 135 & 31 & $(27,36)$ \\
\hline $\mathrm{T} 1-\mathrm{T} 12$ & 160 & 56 & 35 & $(28,43)$ & 47 & 29 & $(22,37)$ \\
\hline $\begin{array}{l}\text { L1-S5 or Cauda } \\
\text { Equina }\end{array}$ & 105 & 10 & 10 & $(5,17)$ & 5 & 5 & $(2,11)$ \\
\hline $\begin{array}{l}\text { C1-T12 + Cauda } \\
\text { Equina }\end{array}$ & 7 & 2 & 29 & $(4,71)$ & 2 & 29 & $(4,71)$ \\
\hline Unknown & 68 & 15 & 22 & $(13,34)$ & 15 & 22 & $(13,34)$ \\
\hline Total & 769 & 246 & 32 & $(29,35)$ & 204 & 27 & $(23,30)$ \\
\hline \multicolumn{8}{|c|}{ Neurological grade (AIS) } \\
\hline A & 174 & 74 & 43 & $(35,50)$ & 63 & 36 & $(29,44)$ \\
\hline B & 68 & 33 & 49 & $(36,61)$ & 29 & 43 & $(31,55)$ \\
\hline $\mathrm{C}$ & 83 & 38 & 46 & $(35,57)$ & 34 & 41 & $(30,52)$ \\
\hline $\mathrm{D}$ & 360 & 84 & 23 & $(19,28)$ & 61 & 17 & $(13,21)$ \\
\hline $\mathrm{E}$ & 15 & 1 & 7 & $(0.2,32)$ & 1 & 7 & $(0.2,32)$ \\
\hline Unknown & 69 & 16 & 23 & $(14,35)$ & 16 & 23 & $(14,35)$ \\
\hline Total & 769 & 246 & 32 & $(29,35)$ & 204 & 27 & $(23,30)$ \\
\hline
\end{tabular}


Table 3 Anti-spasticity medication use listed by medication type

\begin{tabular}{|c|c|c|c|c|c|c|c|c|c|c|c|c|c|c|c|}
\hline \multirow[b]{2}{*}{ Drug } & \multicolumn{2}{|c|}{ Ever } & \multicolumn{2}{|c|}{$\begin{array}{l}\text { At } \\
\text { dis- } \\
\text { charge }\end{array}$} & \multicolumn{3}{|c|}{ Start dose } & \multicolumn{3}{|c|}{ Discharge dose } & \multicolumn{5}{|c|}{ Line of therapy } \\
\hline & $N$ & $\%$ & $N$ & $\%$ & Min & Med & $\operatorname{Max}$ & Min & Med & $\operatorname{Max}$ & $1 \mathrm{st}$ & 2nd & 3 rd & 4th & 5th \\
\hline Baclofen & 239 & 31 & 193 & 25 & 2.5 & 15 & 45 & 2.5 & 30 & 120 & $94 \%$ & $6 \%$ & - & - & - \\
\hline Tizanidine & 38 & 5 & 23 & 3 & 1 & 2 & 24 & 1 & 6 & 26 & - & $89 \%$ & $11 \%$ & - & - \\
\hline Diazepam & 19 & 2 & 12 & 2 & 1 & 2.5 & 16 & 2.5 & 5 & 14 & $10 \%$ & $45 \%$ & $35 \%$ & $10 \%$ & - \\
\hline Clonazepam & 15 & 2 & 3 & 0.4 & 0.25 & 0.5 & 2 & 0.5 & 0.75 & 4 & $53 \%$ & $47 \%$ & - & - & - \\
\hline Dantrolene & 2 & 0.3 & 0 & 0 & 25 & 37.5 & 50 & - & - & - & - & - & $100 \%$ & - & - \\
\hline $\begin{array}{l}\text { Phenol } \\
\text { (Injectable) }\end{array}$ & 1 & 0.1 & 0 & 0 & 10 & 10 & 10 & - & - & - & - & - & - & - & $100 \%$ \\
\hline $\begin{array}{l}\text { Botulinum } \\
\text { toxin } \\
\text { (Injectable) }\end{array}$ & 48 & 6 & 37 & 5 & 50 & 200 & 600 & 100 & 200 & 600 & $23 \%$ & $48 \%$ & $21 \%$ & $8 \%$ & - \\
\hline Oral & 241 & 31 & 199 & 26 & - & - & - & - & - & - & - & - & - & - & - \\
\hline $\begin{array}{l}\text { Oral or } \\
\text { Injectable }\end{array}$ & 246 & 32 & 204 & 27 & - & - & - & - & - & - & - & - & - & - & - \\
\hline
\end{tabular}

$92 \%$ of individuals treated with an anti-spasticity medication and the first oral anti-spasticity medication used in $96 \%$ of the individuals treated with any oral anti-spasticity medication. The most common second line of therapy was Tizanidine with 34 (39\%) individuals receiving Tizanidine as a second-line therapy among $87 \mathrm{~s}$ line prescriptions. Botulinum toxin was the most common third and fourth line therapy, with 10 individuals receiving botulinum toxin (43\%) among 23 third-line prescriptions and 4 individuals receiving botulinum toxin $(67 \%)$ among 6 fourth line prescriptions, respectively.

Botulinum toxin was the most commonly used injectable focal chemodenervation agent, $N=48$. Mean time from injury to first botulinum toxin injection was 141 (66) days. Of the individuals that received botulinum toxin, $31 \%$ had at least one subsequent injection during their inpatient stay. Phenol was used in one patient with $\mathrm{C} 1-\mathrm{C} 8$ neurological level and AIS D at discharge.

The probability of starting any medication (oral or injectable) increased as individuals' length of in-patient stay in either the acute or rehabilitation setting increased, (Fig. 2). The Kaplan-Meier curve in Fig. 2 depicts the estimated probability of having initiated an in-patient antispasticity medication at a given time point post-injury. The number of individuals still in inpatient care at each time point in the data is presented at the bottom of the graph. At 2 months post-injury individuals in in-patient care $(N=$ $472)$ had a $24 \%$ chance $(95 \%$ CI $(20,27))$ of having initiated anti-spasticity medication. This probability increased to $43 \%(95 \% \mathrm{CI}(38,48))$ at 4 months post-injury $(N=280)$, to $55 \%(95 \% \mathrm{CI}(49,60))$ at 6 months post-injury $(N=$ $152)$, and to $71 \%(95 \% \mathrm{CI}(62,79))$ at 12 months postinjury $(N=16)$. By 13 months post-injury, there was $>75 \%$ chance that an inpatient had initiated an anti-spasticity medication during their inpatient stay. There was no evidence of a difference in time to initiation of anti-spasticity medication by AIS, however there was evidence that time to initiation was different by neurological level $(p<0.0001)$. At 75 days post-injury individuals with L1-S5 or cauda equina were significantly less likely to have initiated anti-spasticity medication compared to individuals with $\mathrm{C} 1-\mathrm{C} 8$ injuries.

\section{Anti-spasticity medication use at community discharge}

At discharge from hospital into the community (i.e., community discharge), $27 \%(95 \%$ CI $(23,30))$ of individuals were on anti-spasticity medication (Table 2). Neurological level and AIS grade were associated with individuals being discharged from inpatient care on anti-spasticity medication ( $p<0.0001$ for both), where individuals with cervical or thoracic injury were more likely to be discharged on antispasticity medication compared to individuals with lumbosacral injuries $(p<0.0001)$. Individuals with AIS A, B or C grades were more likely to be discharged on anti-spasticity medication compared to individuals with AIS D grade injuries $(p<0.0001)$.

At community discharge $N=152(20 \%)$ of individuals were on monotherapy, $N=45(6 \%)$ on dual therapy, $N=7$ (1\%) on triple therapy and $N=2$ on quad therapy. The most frequently prescribed combination of anti-spasticity medication at discharge was Baclofen and botulinum toxin injection (4\%). The relative risk of being discharged on an anti-spasticity medication was lower for individuals that initiated spasticity medication within 6 months of injury compared to individuals who initiated treatment $<6$ months post-injury (RR: $0.88,95 \%$ CI $(0.76,1.02))$. There was no 


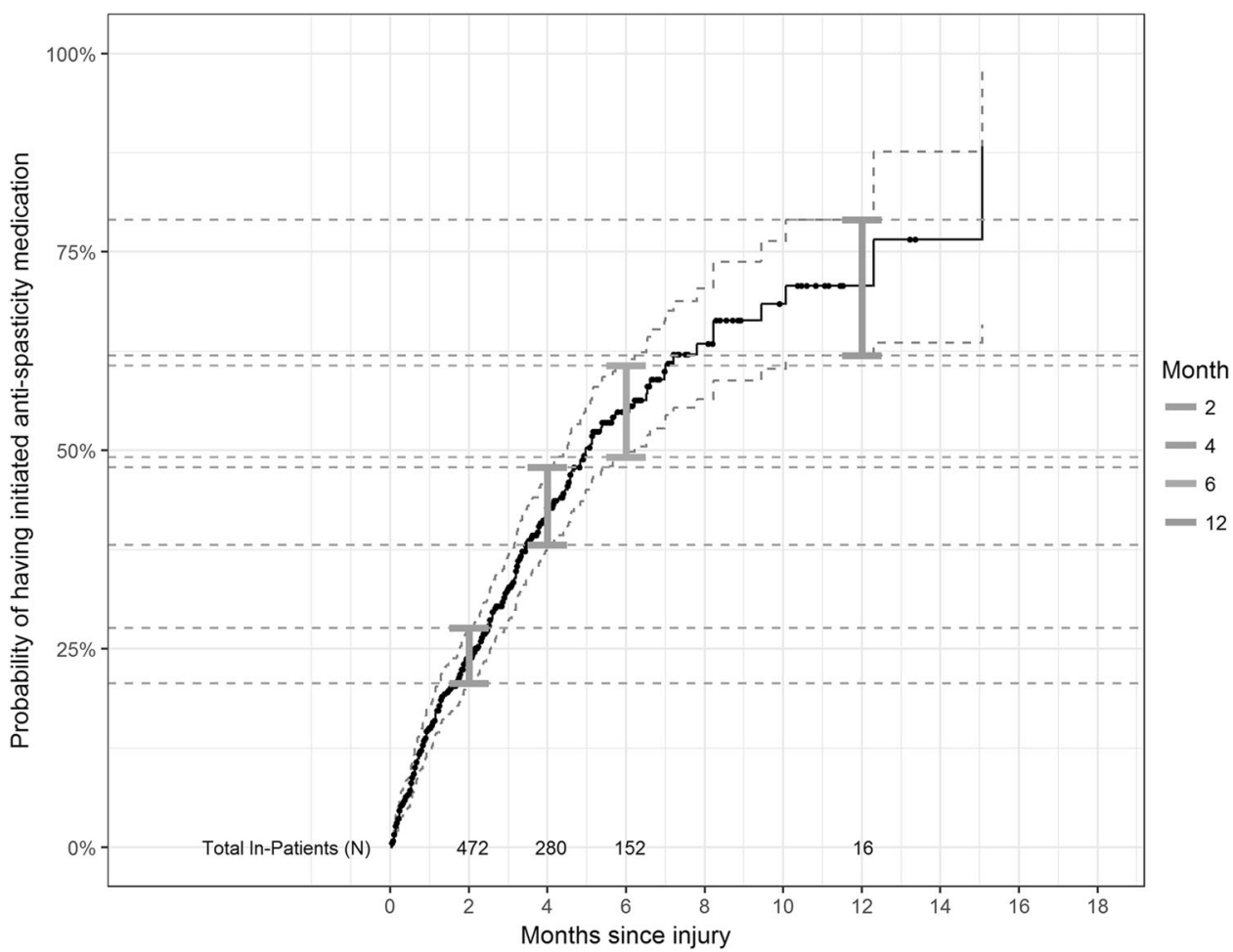

Fig. 2 Probability of having initiated anti-spasticity medication over time

effect of age $(p=0.29)$ or gender $(p=0.14)$ on taking an anti-spasticity medication at discharge.

\section{Practice patterns over time}

Because there were no differences in proportion of individuals on oral or injectable anti-spasticity medication over time (between 2005 and 2014), individuals were grouped into two cohorts for comparative analysis, 2005-2009 and 2010-2014. Results demonstrated that practice patterns have remained stable since 2005 . There is a non-significant trend suggesting lower oral and injectable anti-spasticity medication use over time, $30 \%$ vs. $24 \%(p=0.07)$. There were no differences in proportion of individuals on Baclofen, botulinum toxin or any anti-spasticity medication by neurological level or AIS grade over time.

\section{Discussion}

This is the first study reporting the in-patient treatment pattern of anti-spasticity medication in a large cohort of individuals with traumatic SCI. We found that $32 \%$ of all individuals were prescribed anti-spasticity medication at some point during their hospital admission and $27 \%$ were discharged with an anti-spasticity medication prescription. Our results are consistent with Maynard et al. [4] who reported $37 \%$ of individuals with SCI had received anti- spasticity medication within the first year of injury and 26\% of individuals with chronic SCI were receiving antispasticity medication in the community. Similarly, in a cross sectional analysis Sköld et al. [6] found $28 \%$ of individuals with SCI with reported anti-spasticity medication use. Our results are consistent with smaller studies confirming the prevalence of spasticity requiring medications in roughly one quarter to one third of all individuals with SCI discharged to the community.

Our study expands on previously published results by reporting the timeline of anti-spasticity medication use in the inpatient setting. The median time to Baclofen prescription was 50 days. This finding is consistent with clinical observation that spasticity develops after resolution of spinal shock following SCI [22, 23], and typically becomes problematic in the subacute phase post-SCI [2, 24]. However, it is important to note that anti-spasticity medications were started as early as one day post-injury, suggesting that there is the need for early monitoring for spasticity development. Baclofen was also started as late as 458 days post-injury, which provides support for ongoing spasticity screening after SCI particularly with the trend towards decreasing inpatient lengths of stay, as the need for initiating a spasticity treatment may occur in the outpatient setting where there can be less access to physicians with experience in spasticity management. Based on our results, individuals will most likely to benefit from referral to a specialized physician for spasticity 
monitoring on community discharge are those with neurological levels between $\mathrm{C} 1$ and $\mathrm{T} 12$ (e.g., injuries above the conus) and AIS grades A, B, and C. Individuals with greater motor function (AIS D injuries) at discharge had significantly lower rates of anti-spasticity medication use, consistent with our previous finding that they report lower rates of spasticity [2].

Baclofen has been previously reported as being the most frequently prescribed treatment for spasticity following SCI $[3,24-26]$. We also found that Baclofen was most commonly prescribed as first line therapy, accounting for $92 \%$ of all first line prescriptions and $96 \%$ of first line oral prescriptions. Tizanidine was the most commonly prescribed second line oral therapy. One third of all individuals with SCI were prescribed anti-spasticity medication during inpatient hospital stay and a large percentage $(83 \%)$ of those individuals remained on medication at discharge. This is helpful information for hospital based care teams preparing to transition individuals to community and important handover for primary care providers assuming the medical management of chronic SCI.

The relative risk of being discharged on an anti-spasticity medication was lower for individuals that initiated spasticity medication within 6 months of injury compared to individuals who initiated treatment $>6$ months post-injury. Earlier spasticity treatment may result in the development of less severe spasticity that is eventually amenable to medication discontinuation. Conversely, earlier spasticity treatment may result in unwanted medication side effects leading to discontinuation of the medication which may be more likely to occur in the inpatient setting where individuals are monitored closely. Recent evidence suggests a negative association between anti-spasticity medications and motor recovery [24, 27]. Further investigation into the timing of anti-spasticity medication and its effect on both physical and non-physical domains following SCI is warranted.

Like Priebe et al. [25] and Veerakumar et al. [26] we found maximal discharge doses of Baclofen exceeded the industry recommended dosing of $80 \mathrm{mg} /$ day, with a maximum dose of $120 \mathrm{mg} /$ day. In addition, the maximum dose of botulinum toxin we reported exceeds the recommended industry standard of 400 units for focal spasticity. Whether or not doses above those recommended provide additional benefit with an acceptable risk profile is yet to be determined in this population and also merits further investigation.

At discharge, $20 \%$ of individuals were on monotherapy, $6 \%$ on dual therapy and $1 \%$ on triple therapy. Our findings indicate a lower prevalence for multiple medications use than previously reported; however, our cohort was prospectively enrolled with less potential for selection bias that can occur with recruitment from a pool of individuals with established spasticity as inclusion criteria [19]. Our results probably reflect the true population prevalence of antispasticity medication use following SCI.

Botulinum toxin is a treatment option for focal spasticity management following SCI [28]. The prevalence of its use in the population of individuals with SCI has not been well established. We found that $6 \%$ of our cohort received at least one botulinum toxin injection during inpatient admission. It was used as second line therapy in $48 \%$ of its prescriptions. Like Baclofen, the maximal doses of botulinum toxin in our study also exceeded recommended industry dosing. Botulinum toxin is considered a first line treatment for spasticity post-stroke [29]; however, the pattern of spasticity from spinal origin differs significantly from patterns arising from cerebral origin and further research into the role of botulinum toxin for spasticity management specifically in the SCI population is needed.

\section{Study limitations}

Our study is limited by the strength of the electronic medical record system from which medication data was abstracted. Individuals whose medical records were truncated $(N=90)$ because they exceed storage capacity were not included in the analysis and so estimates of the proportion of individuals on medications may be underestimated. As individuals who were transferred to Vancouver General Hospital and GF Strong Rehab Centre from outside facilities were more likely to have incomplete data and therefore be excluded from the study, these results primarily reflect the treatment patterns of physicians working in the quaternary trauma centre in Vancouver, British Columbia, Canada.

We observed that in-patient anti-spasticity medication use has remained stable since 2004. There was a trend toward lower "traditional" anti-spasticity medication use in 2010-2014 vs. 2005-2009 which may be explained by the rise of atypical anti-spasticity medications that we did not capture including cannabinoids and gabapentin $[16,18]$ as it was difficult to determine if the prescriptions were for neuropathic pain or spasticity. We also did not report if individuals were prescribed intrathecal baclofen which could lower the prevalence of oral anti-spasticity medication use but typically in our centre individuals are not implanted with an intrathecal Baclofen pump until after community discharge. While the nature of the prescribing physician's level of knowledge, training and preferences may influence medication prescriptions and potentially introduce bias, at our center there is a mix of trainees (medical students and resident physicians) and staff (Nurse Practitioners, Hospitalists, Physiatrists, Pain Medicine Specialists, and Orthopedic Surgeons). This model has not 
changed significantly in the last 20 years and it is our opinion that the model of care does not explain the trend toward lower anti-spasticity medication use.

Lastly, clinical measures of spasticity were not evaluated in our study. Conclusions regarding medication use for mild, moderate or severe spasms cannot be drawn from this dataset. Nor do we have any information if medication use resulted in a clinical change in spasm severity. While we acknowledge reporting clinical spasm severity would provide some context to the data, in the RHSCIR database spasm severity was not collected at time of medication initiation or stoppage.

\section{Conclusions}

Approximately one third of all individuals with SCI were prescribed anti-spasticity medication during their acute and rehabilitation hospitalization. Higher anti-spasticity medication use was seen in individuals with cervical and thoracic level injuries compared to individuals with lumbosacral injuries, and AIS A, B, and C grades compared to D grade injuries. This probability of starting anti-spasticity medication increased significantly with time and $70 \%$ of individuals with SCI at 12 months post-injury were prescribed anti-spasticity medication during their hospital admission. Baclofen and Tizanidine were the most common first and second line oral agents and at discharge and some prescriptions exceeded recommended doses. Baclofen was initiated between 1 and 458 days post-injury, supporting early and ongoing screening for spasticity beyond the first year following SCI. Results inform clinicians and individuals of "real-world" spasticity treatment patterns and more research is needed to evaluate the use of both oral and injectable anti-spasticity treatment in the SCI population.

Acknowledgements We are grateful to the following individuals for their contribution: Juliet Batke and Allan Aludino of the Vancouver Spine Research Program for database management and data abstraction; Glenys MacIsaac of the Rick Hansen Institute for technical support of data management; and Darby Thompson and Rachel Lipson from Emmes Canada for statistical support and assistance with data analysis.

Funding Dr. Kwon is the Canada Research Chair in Spinal Cord Injury.

Author contributions $\mathrm{KH}$ was responsible for designing the research protocol, data collection, data interpretation, generated the reference list and was the primary author of the manuscript. ES contributed to research design, performed statistical analysis, contributed to writing the manuscript and created the tables and figure. VN provided feedback on the study design and manuscript. BK provided feedback on the study design and manuscript. PM was responsible for designing the research protocol, assisted in data interpretation and contributed to writing the manuscript.

\section{Compliance with ethical standards}

Conflict of interest Ms. Szefer is an employee of Emmes Canada. Dr. Noonan is an employee of the Rick Hansen Institute. The remaining authors declares no conflict of interest.

\section{References}

1. Pandyan AD, Gregoric M, Barnes MP, Wood D, Van Wijck F, Burridge J, et al. Spasticity: clinical perceptions, neurological realities and meaningful measurement. Disabil Rehabil. 2005;27:2-6.

2. Holtz KA, Lipson R, Noonan VK, Kwon BK, Mills PB. Prevalence and effect of problematic spasticity after traumatic spinal cord injury. Arch Phys Med Rehabil. 2017;98:1132-8.

3. Andresen SR, Biering-Sørensen F, Hagen EM, Nielsen JF, Bach FW, Finnerup NB, et al. Pain, spasticity and quality of life in individuals with traumatic spinal cord injury in Denmark. Spinal Cord. 2016;54:973-9.

4. Maynard FM, Karunas RS, Waring WP. Epidemiology of spasticity following traumatic spinal cord injury. Arch Phys Med Rehabil. 1990;71:566-9.

5. Dvorak MF, Fisher CG, Hoekema J, Boyd M, Noonan V, Wing $\mathrm{PC}$, et al. Factors predicting motor recovery and functional outcome after traumatic central cord syndrome: a long-term followup. Spine. 2005;30:2303-11.

6. Sköld C, Levi R, Seiger A. Spasticity after traumatic spinal cord injury: nature, severity, and location. Arch Phys Med Rehabil. 1999;80:1548-57.

7. Little JWMP, Micklesen, PB Umlauf, RP Britell, CM Lower. Extremity manifestations of spasticity in chronic spinal cord injury. Am J Phys Med Rehabil. 1989;68:32-6.

8. McKay WB, Sweatman WM, Field-Fote EC. The experience of spasticity after spinal cord injury: perceived characteristics and impact on daily life. Spinal Cord. 2018;56:478-86.

9. Sweet SN, Noreau L, Leblond J, Dumont FS. Understanding quality of life in adults with spinal cord injury via SCI-related needs and secondary complications. Top Spinal Cord Inj Rehabil. 2014;20:321-8.

10. Anson CA, Shepherd C. Incidence of secondary complications in spinal cord injury. Int J Rehabil Res. 1996;19:55-66.

11. Fleuren JF, Voerman GE, Snoek GJ, Nene AV, Rietman JS, Hermens HJ, et al. Perception of lower limb spasticity in patients with spinal cord injury. Spinal Cord. 2009;47:396-400.

12. Westerkam D, Saunders LL, Krause JS. Association of spasticity and life satisfaction after spinal cord injury. Spinal Cord. 2011;49:990-4.

13. Elbasiouny SM, Moroz D, Bakr MM, Mushahwar VK. Management of spasticity after spinal cord injury: current techniques and future directions. Neurorehabil Neural Repair. 2010;24:23-33.

14. Lui J, Sarai M, Mills PB. Chemodenervation for treatment of limb spasticity following spinal cord injury: a systematic review. Spinal Cord. 2015;53:252-64.

15. Gormley ME Jr., O’Brien CF, Yablon SA. A clinical overview of treatment decisions in the management of spasticity. Muscle Nerve. 1997;20:14-20.

16. Thompson AJ, Jarrett L, Lockley L, Marsden J, Stevenson VL. Clinical management of spasticity. J Neurol Neurosurg Psychiatry. 2005;76:459-63.

17. Ward AB. A summary of spasticity management-a treatment algorithm. Eur J Neurol. 2002;9:48-52. 
18. Taricco M, Adone R, Pagliacci C, Telaro E. Pharmacological interventions for spasticity following spinal cord injury. Cochrane Database Syst Rev. 2000;2:CD001131.

19. Noonan VK, Kwon BK, Soril L, Fehlings MG, Hurlbert RJ, Townson A, et al. The Rick Hansen Spinal Cord Injury Registry (RHSCIR): A National Patient-registry. Spinal Cord. 2012;50:22-7.

20. Kirshblum SC, Burns SP, Biering-Sorensen F, Donovan W, Graves DE, Jha A, et al. International standards for neurological classification of spinal cord injury. J Spinal Cord Med. 2011;34:535-46.

21. Tilton AH. Injectable neuromuscular blockade in the treatment of spasticity and movement disorders. J Child Neurol. 2003;18 (Suppl 1):S50-66.

22. Dietz V. Spastic movement disorder. Spinal Cord. 2000;38:389-93.

23. Adams MM, Hicks AL. Spasticity after spinal cord injury. Spinal Cord. 2005;43:577-86.

24. Theriault ER, Huang V, Whiteneck G, Dijkers MP, Harel NY. Antispasmodic medications may be associated with reduced recovery during inpatient rehabilitation after traumatic spinal cord injury. J Spinal Cord Med. 2018;41:63-71.

25. Priebe MM, Sherwood AM, Thornby JI, Kharas NF, Markowski J. Clinical assessment of spasticity in spinal cord injury: a multidimensional problem. Arch Phys Med Rehabil. 1996; 77:713-6.

26. Veerakumar A, Cheng JJ, Sunshine A, Ye X, Zorowitz RD, Anderson WS, et al. Baclofen dosage after traumatic spinal cord injury: a multi-decade retrospective analysis. Clin Neurol Neurosurg. 2014;129:50-6.

27. Thomas CK, Hager-Ross CK, Klein CS. Effects of baclofen on motor units paralysed by chronic cervical spinal cord injury. Brain. 2010;133:117-25.

28. Marciniak C, Rader L, Gagnon C. The use of botulinum toxin for spasticity after spinal cord injury. Am J Phys Med Rehabil. 2008;87: 329.

29. Wissel J, Ward A, Erztgaard P, Bensmail D, Hecht M, Lejeune T, et al. European consensus table on the use of botulinum toxin type A in adult spasticity. J Rehabil Med. 2009;41:13-25. 